Pacific Journal of Mathematic 


\title{
CONVERSE MEASURABILITY THEOREMS FOR YEH-WIENER SPACE
}

\author{
Kun Soo Chang
}

\begin{abstract}
Cameron and Storvick established a theorem for evaluating in terms of a Wiener integral the Yeh-Wiener integral of a functional of $x$ which depends on the values of $x$ on a finite number of horizontal lines. Skoug obtained the converse of the theorem in case of one horizontal line. In this paper we extend Skoug's result to the case of a finite number of horizontal lines.
\end{abstract}

1. Introduction. Let $C_{1}[a, b]$ denote the Wiener space of functions of one variable, i.e., $C_{1}[a, b]=\{x(\cdot) \mid x(a)=0$ and $x(s)$ is continuous on $[a, b]\}$. Let $R=\{(s, t) \mid a \leqq s \leqq b, \alpha \leqq t \leqq \beta\}$ and let $C_{2}[R]$ be YehWiener space (or 2 parameter Wiener space), i.e., $C_{2}[R]=\{x(\cdot, \cdot) \mid x(a, t)=$ $x(s, \alpha)=0, x(s, t)$ is continuous on $R\}$. Let $\nu$ be Wiener measure on $C_{1}[a, b]$ and let $m$ be Yeh-Wiener measure on $C_{2}[R]$. For a discussion of Yeh-Wiener measure see [1], [3] and [4]. $R$ will denote the real numbers and $\boldsymbol{C}$ the complex numbers. We shall use the following notation for the Cartesian product of $n$ Wiener spaces

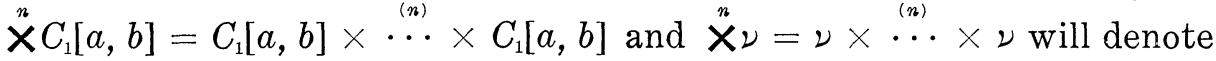
the product of $n$ Wiener measures on $\dot{x}^{n} C_{1}[a, b]$.

Let $\alpha=t_{0}<t_{1}<\cdots<t_{n}=\beta$ be a subdivision of $[\alpha, \beta]$. Define $\varphi: \stackrel{n}{\times} C_{1}[a, b] \rightarrow \stackrel{n}{\times} C_{1}[a, b]$ by

$$
\begin{aligned}
\mathcal{P}\left(y_{1}, y_{2}, \cdots, y_{n}\right) & \\
=\left(\sqrt{\frac{t_{1}-t_{0}}{2}} y_{1}, \sqrt{\frac{t_{1}-t_{0}}{2}} y_{1}\right. & +\sqrt{\frac{t_{2}-t_{1}}{2}} y_{2}, \cdots, \sqrt{\frac{t_{1}-t_{0}}{2}} y_{1}+\cdots \\
& \left.+\sqrt{\frac{t_{n}-t_{n-1}}{2}} y_{n}\right) .
\end{aligned}
$$

Then $\varphi$ is $1-1$, onto and continuous with respect to the uniform topology. Let $G: C_{2}[R] \rightarrow \stackrel{n}{\times} C_{1}[a, b]$ be defined by $G(x)=\left(x\left(\cdot, t_{1}\right), x\left(\cdot, t_{2}\right)\right.$, $\left.\cdots, x\left(\cdot, t_{n}\right)\right)$. Then $G$ is a continuous function from $C_{2}[R]$ onto $\stackrel{n}{\times} C_{1}[a, b]$.

In [1] Cameron and Storvick evaluated certain Yeh-Wiener integrals in terms of Wiener integrals. In particular they obtained the following theorem;

TheOREM A (n-parallel lines theorem). Let $f\left(y_{1}, y_{2}, \cdots, y_{n}\right)$ be a real or complex valued functional defined on $\stackrel{n}{x}^{n}\left[\begin{array}{l}{[} \\ 1\end{array}, b\right]$ such that 
$f \circ \varphi$ is a Wiener measurable functional of $\left(y_{1}, y_{2}, \cdots, y_{n}\right)$ on $\stackrel{n}{\times} C_{1}[a, b]$. Then $f \circ G$ is a Yeh-Wiener measurable functional of $x$ on $C_{2}[R]$ and

$$
\int_{C_{2}[R]} f \circ G(x) d x=\int_{\times C_{1}[a, b]} f \circ \varphi\left(y_{1}, y_{2}, \cdots, y_{n}\right) d\left(y_{1} \times \cdots \times y_{n}\right)
$$

where the existence of either integral implies the existence of the other and their equality.

We note that Theorem $\mathrm{A}$, in the case $n=1$, is called the one line theorem. Now we explicitly state and prove the following corollary of Theorem A which plays a key role in the proof of Lemma 3 in $\S 2$.

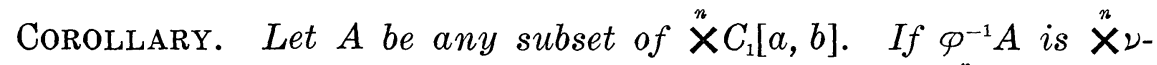
measurable, then $G^{-1} A$ is Yeh-Wiener measurable and ${ }^{n} \nu\left(\varphi^{-1} A\right)=$ $m\left(G^{-1} A\right)$.

$$
\begin{aligned}
& \text { Proof. Let } f\left(y_{1}, y_{2}, \cdots, y_{n}\right)=\chi_{A}\left(y_{1}, y_{2}, \cdots, y_{n}\right) \text {. Then } \\
& f \circ \varphi\left(y_{1}, y_{2}, \cdots, y_{n}\right)=\chi_{A}\left(\varphi\left(y_{1}, y_{2}, \cdots, y_{n}\right)\right)=\chi_{\varphi^{-1} A}\left(y_{1}, y_{2}, \cdots, y_{n}\right) .
\end{aligned}
$$

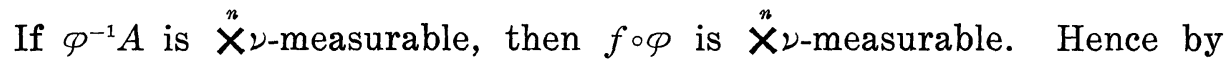
Theorem A, $f \circ G$ is a Yeh-Wiener measurable functional of $x$ on $C_{2}[R]$. But $f \circ G(x)=\chi_{A}(G(x))=\chi_{G^{-1}{ }_{A}}(x)$. Thus $G^{-1} A$ is Yeh-Wiener measurable.

$$
\begin{aligned}
\stackrel{n}{\times} \nu\left(\varphi^{-1} A\right) & =\int_{\times C_{1}[a, b]} \chi_{\varphi^{-1} A}\left(y_{1}, y_{2}, \cdots, y_{n}\right) d\left(y_{1} \times \cdots \times y_{n}\right) \\
& =\int_{\times C_{1}[a, b]} f \circ \varphi\left(y_{1}, y_{2}, \cdots, y_{n}\right) d\left(y_{1} \times \cdots \times y_{n}\right) \\
& =\int_{C_{2}[R]} f\left(x\left(\cdot, t_{1}\right), \cdots, x\left(\cdot, t_{n}\right)\right) d x \\
& =\int_{C_{2}[R]} f \circ G(x) d x=\int_{C_{2}[R]} \chi_{G^{-1} A}(x) d x=m\left(G^{-1} A\right) .
\end{aligned}
$$

It has long been known that measurability questions in Wiener space and Yeh-Wiener space are often rather delicate. In [3] Skoug established some relationships between Yeh-Wiener measurability and Wiener measurability of certain sets and functionals. Furthermore he obtained the converse of the one line theorem. In this paper we extend his result to the $n$-parallel lines theorem. In particular we show that if $A$ is any subset of ${ }^{n} C_{1}[a, b]$, then $G^{-1} A$ is a Yeh-Wiener measurable subset of $C_{2}[R]$ if and only if $\varphi^{-1} A$ is a Wiener measurable subset of $\stackrel{n}{\times} C_{1}[a, b]$.

2. Lemmas. The converse measurability theorems in $\S 3$ will follow quite readily once we establish three lemmas. 
Definition. Let $\delta$ be a fixed constant satisfying $0<\delta<1 / 2$ and let $\lambda>0$ be given. Let

$$
\begin{aligned}
A_{\lambda} \equiv A_{\lambda}(\delta) \equiv & \left\{x \in C_{2}[R]:\left|x\left(s_{2}, t_{2}\right)-x\left(s_{1}, t_{1}\right)\right| \leqq \lambda\left[\left(s_{2}-s_{1}\right)^{2}+\left(t_{2}-t_{1}\right)^{2}\right]^{\delta / 2}\right. \\
& \text { for all } \left.s_{1}, s_{2} \in[a, b] \text { and } t_{1}, t_{2} \in[\alpha, \beta]\right\} .
\end{aligned}
$$

Our first lemma is taken from [3]. We state it without proof.

Lemma 1. (a) For any $\varepsilon>0$, there exists $\lambda_{0}>0$ such that $m\left(A_{\lambda}^{c}\right)<\varepsilon$ for all $\lambda \geqq \lambda_{0}$. In fact $m\left(\mathbf{U}_{n=1}^{\infty} A_{n}\right)=1$. (b) For each $\lambda>0, A_{\lambda}$ is compact in the uniform topology in $C_{2}[R]$.

Lemma 2. Let $A$ be any subset of $\stackrel{n}{\times} C_{1}[a, b]$ and let $V$ be any open set in $C_{2}[R]$ containing $G^{-1} A$. Let $\lambda>0$ be given. Then there exists an open set $U$ in $\stackrel{n}{\times}_{C_{1}}[a, b]$ such that $A \subseteq U$ and $A_{\lambda} \cap G^{-1} U \subseteq V$.

Proof. Case 1. Assume that $A$ consists of just one point, say, $\left(y_{1}, \cdots, y_{n}\right)$. Suppose that Lemma 2 is false. For $n=1,2,3, \cdots$, let $U_{n}$ be open sphere of radius $1 / n$ about $\left(y_{1}, y_{2}, \cdots, y_{n}\right)$. Then there exists a sequence of points $\left\{x_{n}\right\}_{n=1}^{\infty}$ in $\left(A_{2} \cap G^{-1} U_{n}\right) \backslash V$. Hence $\left\{x_{n}\right\}_{n=1}^{\infty} \subseteq$ $A_{\lambda}$ and $\left\|G x_{n}-\left(y_{1}, y_{2}, \cdots, y_{n}\right)\right\|<1 / n$ where $\|\cdot\|$ is a product norm in $\stackrel{n}{x}_{C_{1}}[a, b]$. Since $A_{2}$ is compact in the uniform topology for $C_{2}[R]$, there exists a subsequence $\left\{x_{n_{b}}\right\}_{k=1}^{\infty}$ which converges uniformly on $R$ to some element, say $x_{0}$, of $C_{2}[R]$. By continuity of $G,\left(y_{1}, \cdots, y_{n}\right)=$ $\lim _{k \rightarrow \infty} G x_{n_{k}}=G x_{0}$. Thus $G x_{0}$ is in $A$ and $x_{0}$ is in $G^{-1} A$. But $V^{c}$ is closed and so $x_{0}$ is in $V^{c} \cong\left(G^{-1} A\right)^{c}$ which is contrary to $x_{0} \in G^{-1} A$.

Case 2. General case. Let $A$ be any set in ${ }^{x} C_{1}[a, b]$. By Case 1 , we see that for each point $z$ in $A$ there exists an open set $U_{z}$ in $\stackrel{n}{\times} C_{1}[a, b]$ such that $z \in U_{z}$ and $A_{\lambda} \cap G^{-1} U_{z} \cong V$. Then $U \equiv \mathbf{U}_{z \in A} U_{z}$ is an open set in $\stackrel{n}{\times}^{\prime} C_{1}[a, b]$ containing $A$ and

$$
A_{\lambda} \cap G^{-1} U=A_{\lambda} \cap\left(G^{-1}\left(\bigcup_{z \in A} U_{z}\right)\right)=\bigcup_{z \in A}\left(A_{\lambda} \cap G^{-1} U_{z}\right) \subseteq V .
$$

Lemma 3. Let $A$ be any subset of $\stackrel{n}{\times}^{n}[a, b]$. Then $m^{*} G^{-1} A=$ $\left(\mathscr{X}^{n} \nu\right)^{*}\left(\varphi^{-1} A\right)$ where $m^{*}$ and $\left(\mathscr{X}^{n} \nu\right) *$ are outer Yeh-Wiener and product Wiener measures respectively.

Proof. First we will show that $m^{*} G^{-1} A \leqq(\stackrel{n}{\times} \nu)^{*}\left(\varphi^{-1} A\right)$. Let $\widetilde{A}$

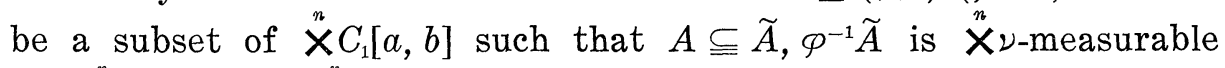
and $\mathscr{X}^{n} \nu\left(\varphi^{-1} \tilde{A}\right)=\left(\mathscr{X}^{n} \nu\right)^{*}\left(\varphi^{-1} A\right)$. Note that such $\hat{A}$ exists since there

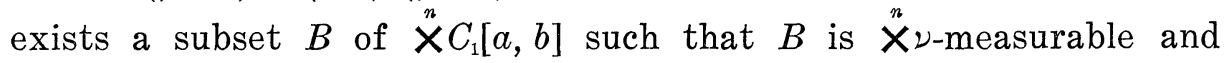


$\stackrel{n}{X}^{\prime}(B)=(\stackrel{n}{\times} \nu)^{*}\left(\varphi^{-1} A\right)$ and $\varphi^{-1} A \subseteq B$. Let $\tilde{A}=\varphi(B)$. Then $A=$ $\varphi\left(\varphi^{-1} A\right) \leqq \varphi(B)=\widetilde{A}$ and $\varphi^{-1}(\widetilde{A})=\varphi^{-1}(\varphi(B))=B$. By Corollary of Theorem A, $G^{-1} \widetilde{A}$ is Yeh-Wiener measurable and $m^{*} G^{-1} A \leqq m^{*} G^{-1} \widetilde{A}=$ $m G^{-1} \tilde{A}=\stackrel{n}{X} \nu\left(\varphi^{-1} \tilde{A}\right)=(\stackrel{n}{X} \nu)^{*}\left(\varphi^{-1} A\right)$.

To show $\left(\mathscr{X}^{n} \nu\right)^{*}\left(\varphi^{-1} A\right) \leqq m^{*} G^{-1} A$, it suffices to show that for given $\varepsilon>0,\left(\dot{X}^{n} \nu\right)^{*}\left(\varphi^{-1} A\right) \leqq m^{*} G^{-1} A+\varepsilon$. Now choose a Yeh-Wiener measurable set $H$ such that $G^{-1} A \subseteq H$ and $m^{*} G^{-1} A=m H$. Next we choose $n>0$ so large that $m\left(A_{n}^{c}\right)<\varepsilon / 2$ [Lemma 1]. Then

$$
m\left(H \cup A_{n}^{c}\right) \leqq m H+m\left(A_{n}^{c}\right)<m^{*} G^{-1} A+\varepsilon / 2 .
$$

Let $V$ be an open subset of $C_{2}[R]$ such that $H \cup A_{n}^{\prime} \subseteq V$ and $m\left(V \backslash\left[H \cup A_{n}^{c}\right]\right)<\varepsilon / 2[2$, Theorem 1.2, p. 27]. Then

$$
m V<m\left(H \cup A_{n}^{c}\right)+\varepsilon / 2 .
$$

By Lemma 2 (note that $G^{-1} A \subseteq H \subseteq H \cup A_{n}^{c} \subseteq V$ and $V$ is open), there exists an open set $U \subseteq \mathscr{x}^{n} C_{1}[a, b]$ such that $A \subseteq U$ and $A_{n} \cap G^{-1} U \subseteq$ $V$. But $\left(G^{-1} U\right) \cap A_{n}^{c} \subseteq A_{n}^{c} \subseteq H \cup A_{n}^{c} \subseteq V$. Hence

$$
G^{-1} U=\left(G^{-1} U \cap A_{n}\right) \cup\left(G^{-1} U \cap A_{n}^{c}\right) \subseteq V .
$$

Since $U$ is open and $\varphi$ is continuous, $\varphi^{-1} U$ is open. Hence $\varphi^{-1} U$ is $\stackrel{n}{X}^{\nu}$-measurable. By continuity of $G, G^{-1} U$ is Yeh-Wiener measurable

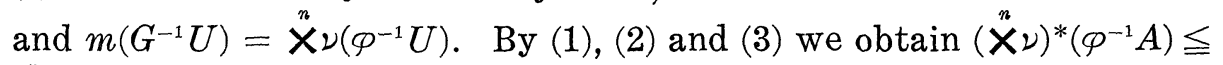
$\stackrel{n}{\times} \nu\left(\varphi^{-1} U\right)=m\left(G^{-1} U\right) \leqq m V<m\left(H \cup A_{n}^{o}\right)+\varepsilon / 2<m^{*} G^{-1} A+\varepsilon$.

3. Converse measurability theorems. Our first theorem in this section establishes a relationship between Yeh-Wiener measurability and product Wiener measurability of certain related sets. In Theorem 2 we obtain the converse of Theorem A.

Theorem 1. Let $A$ be any subset of $\stackrel{n}{x}^{\prime} C_{1}[a, b]$. Then $G^{-1} A$ is Yeh-Wiener measurable if and only if $\varphi^{-1} A$ is $\times^{n} \nu$-measurable. Furthermore $m\left(G^{-1} A\right)=\stackrel{n}{\times} \nu\left(\varphi^{-1} A\right)$.

Proof. We only need to show that if $G^{-1} A$ is Yeh-Wiener measurable then $\varphi^{-1} A$ is $\stackrel{n}{X}_{\nu}$-measurable. So assume that $G^{-1} A$ is Yeh-Wiener measurable. By Lemma $3, m^{*} G^{-1} A=\left(\dot{X}^{n} \nu\right)^{*}\left(\varphi^{-1} A\right)$. Another application of Lemma 3 yields $\left(\stackrel{x}{X} \nu^{*}\right)^{*}\left(\varphi^{-1} A\right)^{c}=\left(\dot{X}^{n} \nu\right)^{*}\left(\varphi^{-1} A^{c}\right)=m^{*} G^{-1} A^{c}=$ $m^{*}\left(G^{-1} A\right)^{c}=m\left(G^{-1} A\right)^{c}$. Thus we obtain that

$$
\begin{aligned}
\left(\stackrel{n}{X}^{\prime} \nu\right)^{*}\left(\varphi^{-1} A\right)^{c}+(\stackrel{n}{\mathbf{X}} \nu)^{*}\left(\varphi^{-1} A\right) & =m^{*}\left(G^{-1} A\right)^{c}+m^{*}\left(G^{-1} A\right) \\
& =m\left(G^{-1} A\right)+m\left(G^{-1} A\right)^{c}=1
\end{aligned}
$$


from which it follows that $\varphi^{-1} A$ is $\stackrel{n}{X}^{n}$-measurable.

THEOREM 2. Let $\alpha=t_{0}<t_{1}<\cdots<t_{n}=\beta$ and let $f\left(y_{1}, y_{2}, \cdots, y_{n}\right)$ be a real or complex valued functional defined on ${ }^{n} C_{1}[a, b]$. Then $f \circ \varphi$ is a Wiener measurable functional of $\left(y_{1}, y_{2}, \cdots, y_{n}\right)$ on $\times C_{1}[a, b]$ if and only if $f \circ G$ is a Yeh-Wiener measurable functional of $x$ on $C_{2}[R]$. In this case,

$$
\int_{C_{2}[R]} f \circ G(x) d x=\int_{\times C_{1}[a, b]} f \circ \phi\left(y_{1}, y_{2}, \cdots, y_{n}\right) d\left(y_{1} \times \cdots \times y_{n}\right)
$$

where the existence of either integral implies the existence of the other and their equality.

Proof. By Theorem A it suffices to show measurability only. Let $B$ be any Borel set in $\boldsymbol{R}$ or $C$. Suppose that $f \circ G$ is Yeh-Wiener measurable. Then $G^{-1}\left(f^{-1} B\right)=(f \circ G)^{-1}(B)$ is Yeh-Wiener measurable. By Theorem $1, \varphi^{-1}\left(f^{-1} B\right)=(f \circ \varphi)^{-1}(B)$ is $\mathscr{X}^{n} \nu$-measurable. Hence $f \circ \varphi$ is ${ }^{n} \nu$-measurable.

\section{REFERENCES}

1. R. H. Cameron and D. A. Storvick, Two related integrals over spaces of continuous functions, Pacific J. Math., 55 (1974), 19-37.

2. K. R. Parthasarathy, Probability Measures on Metric Space, Academic Press, New York, 1967.

3. D. L. Skoug, Converses to measurability theorems for Yeh-Wiener space, Proc. Amer. Math. Soc., 57 (1976), 304-310.

4. J. Yeh, Wiener measure in a space of functions of two variables, Trans. Amer. Math. Soc., 95 (1960), 433-450.

Received November 11, 1980. The author wishes to thank Professor G. W. Johnson for his advice in the writing of this paper.

YONSEI UNIVERSITY

SEOUL, KOREA 



\section{PACIFIC JOURNAL OF MATHEMATICS}

\section{EDITORS}

DONALD BABBITT (Managing Editor)

University of California

Los Angeles, California 90024

Hugo RossI

University of Utah

Salt Lake City, UT 84112

C. C. MOORE and ANDREW OGG

University of California

Berkeley, CA 94720

\section{J. DugundJI}

Department of Mathematics University of Southern California Los Angeles, California 90007

R. Finn and J. Milgram Stanford University

Stanford, California 94305

\section{ASSOCIATE EDITORS}
R. ARENS
E. F. BeCKENBACH
B. H. Neumann
F. WoLF
K. YoSHIDA

\section{SUPPORTING INSTITUTIONS}

UNIVERSITY OF ARIZONA

UNIVERSITY OF BRITISH COLUMBIA

CALIFORNIA INSTITUTE OF TECHNOLOGY

UNIVERSITY OF CALIFORNIA

MONTANA STATE UNIVERSITY

UNIVERSITY OF NEVADA, RENO

NEW MEXICO STATE UNIVERSITY

OREGON STATE UNIVERSITY
UNIVERSITY OF OREGON

UNIVERSITY OF SOUTHERN CALIFORNIA

STANFORD UNIVERSITY

UNIVERSITY OF HAWAII

UNIVERSITY OF TOKYO

UNIVERSITY OF UTAH

WASHINGTON STATE UNIVERSITY

UNIVERSITY OF WASHINGTON 


\section{Pacific Journal of Mathematics}

\section{Vol. 97, No. $1 \quad$ January, 1981}

Charles A. Asmuth and Joe Repka, Tensor products for $S L_{2}(\mathfrak{k})$. II.

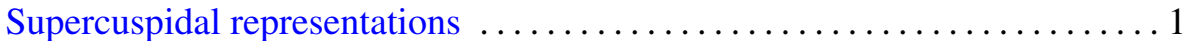

Joseph Barback, On finite sums of regressive isols . ................. 19

Matthew G. Brin and Daniel Russell McMillan, Jr., Generalized

three-manifolds with zero-dimensional nonmanifold set ............29

Kun Soo Chang, Converse measurability theorems for Yeh-Wiener space . . . 59

Christopher Brian Croke, A "maximal torus" type theorem for complete

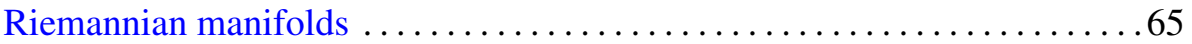

Gustave Adam Efroymson, Sums of squares in planar Nash rings . . . . . . 75

John Robert Fisher, Axiomatic radical and semisimple classes of rings . . . .81

Betty Kvarda, Consecutive integers for which $n^{2}+1$ is composite .......93

Roosevelt Gentry, New diagram proofs of the Hausdorff-Young theorem

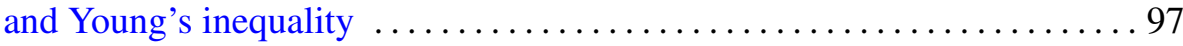

Patrick M. Gilmer, Topological proof of the $G$-signature theorem for $G$

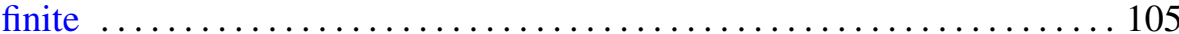

Chung Wei Ha, A noncompact minimax theorem .................. 115

James J. Hebda, Manifolds admitting taut hyperspheres ................ 119

Takayuki Kawada, Sample functions of Pólya processes ............. 125

Peter K. F. Kuhfittig, Common fixed points of nonexpansive mappings by

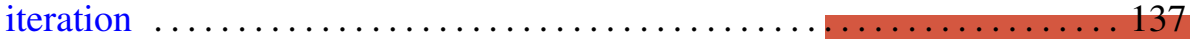

James Thomas Loats and Judith Roitman, Almost rigid Hopfian and dual

Hopfian atomic Boolean algebras .......................... 141

Roger McCann, On embedding semiflows into a radial flow on $l_{2} \ldots \ldots \ldots 151$

John McDonald, Closed orbits of convex sets of operators on the disk

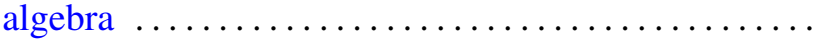

Mark D. Meyerson, Convexity and the table theorem .............. 167

Arnold William Miller, Generic Souslin sets . . . . . . . . . . . . . . . . 171

Takemi Mizokami, On the closed images of paracomplexes $\ldots \ldots \ldots \ldots \ldots 3$

Jagannadham Venkata Pakala and Thomas Stephen Shores, On

compactly packed rings $\ldots \ldots \ldots \ldots \ldots \ldots \ldots \ldots \ldots$

Andrew Pletch, Strong completeness in profinite groups

Wilbur Carrington Whitten, Inverting double knots

James Juei-Chin Yeh, Existence of strong solutions for stochastic

differential equations in the plane 